\title{
Electrical analogous in viscoelasticity
}

\author{
Guido Ala ${ }^{\mathrm{a}, *}$, Mario Di Paola ${ }^{\mathrm{b}}$, Elisa Francomano ${ }^{\mathrm{c}}$, Yan $\mathrm{Li}^{\mathrm{d}}$, Francesco P. Pinnola ${ }^{\mathrm{b}}$ \\ ${ }^{a}$ Università degli Studi di Palermo, Dipartimento di Energia, Ingegneria dell'Informazione e Modelli Matematici (DEIM), Viale delle Scienze Ed.9, \\ I-90128 Palermo, Italy \\ ${ }^{\mathrm{b}}$ Università degli Studi di Palermo, Dipartimento di Ingegneria Civile, Ambientale ed Aerospaziale, dei Materiali (DICAM), Viale delle Scienze \\ Ed.8, I-90128 Palermo, Italy \\ ${ }^{\mathrm{c}}$ Università degli Studi di Palermo, Dipartimento di Ingegneria Chimica, Gestionale, Informatica e Meccanica (DICGIM), Viale delle Scienze Ed.6, \\ I-90128 Palermo, Italy \\ ${ }^{\mathrm{d}}$ School of Control Science and Engineering, Shandong University, Jinan, Shandong 250061, PR China
}

\section{A R T I C L E I N F O}

\section{Article history:}

Received 24 July 2013

Received in revised form 30 September 2013

Accepted 11 November 2013

Available online 23 November 2013

\section{Keywords:}

Fractional calculus

Viscoelastic models

Fractional capacitor

Eigenvalues analysis

\begin{abstract}
A B S T R A C T
In this paper, electrical analogous models of fractional hereditary materials are introduced. Based on recent works by the authors, mechanical models of materials viscoelasticity behavior are firstly approached by using fractional mathematical operators. Viscoelastic models have elastic and viscous components which are obtained by combining springs and dashpots. Various arrangements of these elements can be used, and all of these viscoelastic models can be equivalently modeled as electrical circuits, where the spring and dashpot are analogous to the capacitance and resistance, respectively. The proposed models are validated by using modal analysis. Moreover, a comparison with numerical experiments based on finite difference time domain method shows that, for long time simulations, the correct time behavior can be obtained only with modal analysis. The use of electrical analogous in viscoelasticity can better reveal the real behavior of fractional hereditary materials.
\end{abstract}

(c) 2013 Elsevier B.V. All rights reserved.

\section{Introduction}

In the last few decades, fractional calculus has attracted a great interest in various scientific areas including physics and engineering [1-9]. Particularly, in the area of viscoelasticity a significant effort has been done in describing more closely the behavior of materials by using fractional mathematical models. Moreover, the analogy between viscoelastic and electrical constitutive equations is well-known so that, in spite of different physical meanings, the widely used Maxwell model, Kelvin-Voigt model, and Standard Linear Solid Model can be applied to predict a circuit behavior as well [10]. Besides, allow for the time varying distributions of elements, a series of generalized models are proposed in either canonical structure or ladder networks [11,12], such as the Maxwell-Wiechert model. All the above mentioned viscoelastic models have elastic and viscous components which are combined of springs and dashpots. The only difference is the arrangement of these elements, and all of these viscoelastic models can be equivalently modeled as electrical circuits, where the spring and dashpot are analogous to the capacitance and resistance respectively [13-15]. Nevertheless, compare to two viscoelastic elements, there are four passive electrical elements including resistor, capacitor, inductor and the recently find memristor [16,17]. Thus, although the circuits of LC, RC, RL, etc. can be transformed in some circumstances, it is still reasonable to expect that there are far more new properties included in the electrical models that are formulated by using the same structure in viscoelastic

\footnotetext{
* Corresponding author. Tel.: +39 091 23860288; fax: +39 091488452.

E-mail addresses: guido.ala@unipa.it (G. Ala), mario.dipaola@unipa.it (M. Di Paola), elisa.francomano@unipa.it (E. Francomano), liyan.sdu@gmail.com (Y. Li), francesco.pinnola@unipa.it (F.P. Pinnola).
} 
models. Particularly, the introduction of the fractional elements [18] and power-law phenomena cannot only extend the above discussions but also better reveal the real physical world such as the mechanical model of fractional hereditary materials [19] and the Abel's singular problem [20]. In the paper, mechanical models of viscoelasticity behavior are firstly approached by using fractional operators, based on recent works by the authors [19,21,22]. Then, electrical analogous models are introduced in order to obtain electrical equivalent circuits useful to predict the behavior of fractional hereditary materials in an easy way. The validity of the proposed models is demonstrated by using modal analysis. Moreover, the comparison with numerical experiments based on finite difference time domain (FDTD) method shows that, for long time simulations, the correct time behavior can be obtained only with modal analysis.

\section{Mechanical models of fractional viscoelasticity}

Many materials, like rubbers, polymers, bones, bitumen and so on, show a viscoelastic mechanical behavior; moreover also biological tissues have viscoelastic properties [23-28]. Viscoelasticity is the property of such materials that exhibit at the same time elastic and viscous behavior. The elastic behavior is typical of simple solid materials in which the strain history $\gamma(t)$ is linked by the stress history $\sigma(t)$ through a proportional relation as shown in Eq. (1):

$$
\sigma(t)=E \gamma(t)
$$

where $E$ is the Young modulus (Pascal). Eq. (1) shows the so-called Hooke law, the mechanical model of elasticity is represented by a perfect spring with stiffness $E$ as shown in Fig. 1(a). Instead, the viscous behavior is typical of perfect fluid in which there are stress and strain history linked by the Newtonian law as shown in following equation:

$$
\sigma(t)=\eta \frac{d}{d t} \gamma(t)=\eta \dot{\gamma}(t)
$$

where $\eta$ is the viscosity (Poise) of the fluid. In this case the stress history $\sigma(t)$ is related to the rate of deformation $\dot{\gamma}(t)$ and the model that describes this behavior is the dashpot shown in Fig. 1(b).

In order to describe the viscoelastic behavior, the mechanical models of Fig. 1 are inadequate and, over time, some researchers have used several more or less complex assemblies of the two simple elements of Fig. 1, as it was done by Kelvin, Voigt, Maxwell, Zener, etc. [29,30]. In these models the stress-strain relation is described by following relation:

$$
\sum_{k=0}^{n} a_{k} \frac{d^{k}}{d t^{k}} \sigma(t)=\sum_{k=0}^{m} b_{k} \frac{d^{k}}{d t^{k}} \gamma(t)
$$

Another way to describe the time dependent behavior of viscoelastic materials is by the integral formulation. In fact, from the relaxation function $G(t)$, that represents the stress $\sigma(t)$ for an assigned strain history $\gamma(t)=H(t)$ (where $H(t)$ is the unit step function) and by using the Boltzmann superposition integral the following stress-strain relation is obtained:

$$
\sigma(t)=\int_{0}^{t} G(t-\bar{t}) d \gamma(\bar{t})=\int_{0}^{t} G(t-\bar{t}) \dot{\gamma}(\bar{t}) d \bar{t}
$$

Eq. (4) is valid for quiescent system at $t=0$. All classical models, whose constitutive law is described by Eq. (3), have as relaxation function $G(t)$ a function based on an exponential law. Several scientists have experimentally demonstrated that the relaxation function is not well described by an exponential law, but it follows a power-law trend [21,31-34] of the following type:

$$
G(t)=\frac{C(\beta)}{\Gamma(1-\beta)} t^{-\beta}
$$

where $\Gamma(\cdot)$ is the Euler gamma function, $C(\beta)$ and $\beta$ are parameters that depend on the specific material. By using the relaxation function of Eq. (5) and applying the Boltzmann superposition integral of Eq. (4), another stress-strain relation is obtained:

$$
\sigma(t)=C(\beta)\left({ }_{C} D_{0^{+}}^{\beta} \gamma\right)(t)
$$

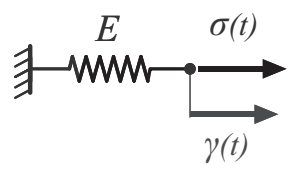

(a) Perfect spring

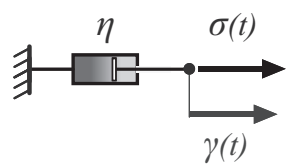

(b) Perfect dashpot

Fig. 1. Elastic and viscous models 
where $\left({ }_{c} D_{0^{+}}^{\beta} \gamma\right)(t)$ is the Caputo's fractional derivative [35-41] of order $\beta$ of strain history $\gamma(t)$ with respect to time $t$, that is defined as

$$
\left({ }_{c} D_{0^{+}}^{\beta} \gamma\right)(t)=\frac{1}{\Gamma(1-\beta)} \int_{0}^{t}(t-\bar{t})^{-\beta} \dot{\gamma}(\bar{t}) d \bar{t}
$$

Eq. (6) shows the stress-strain relation of a new mathematical model of viscoelasticity that is known as spring-pot [42-45]; the involved $\beta$ order of fractional operator is defined in the range ]0,1[ ( $\beta=0$ corresponds to pure elastic behavior of Eq. ( 1 ), while $\beta=1$ represents the pure viscous behavior of Eq. (2)).

The mechanical meaning of the fractional mathematical model reported in Eq. (6) is recently provided by Di Paola \& Zingales [22]. In particular the authors have distinguished two cases of viscoelasticity, one for the behavior in which the elastic phase predominates (Elasto-Viscous materials $0<\beta<1 / 2$ ) and the other in which viscous phase is predominant (ViscoElastic materials $1 / 2<\beta<1)$.

For the Elasto-Viscous (EV) materials the exact mechanical model is a massless indefinite fluid column resting on a bed of independent springs as shown in Fig. 2(a), while for the Visco-Elastic (VE) materials the exact mechanical model is a massless indefinite shear-type column resting on a bed of independent dashpot as shown in Fig. 2(b).

By introducing a $z$ vertical axis, both for the EV model and for the VE model, the fractional stress-strain relation of Eq. (6) is obtained at the top of the lamina if, as stiffness coefficient $k_{i}(z)$ and dashpot coefficient $c_{i}(z)(i=E$ for the EV model and $i=V$ for VE model) the following expressions are considered:

$$
\begin{aligned}
& (\mathrm{EV})\left\{\begin{array}{l}
k_{E}(z)=\frac{G_{0}}{\Gamma(1+\alpha)} z^{-\alpha}, \\
c_{E}(z)=\frac{\eta_{0}}{\Gamma(1-\alpha)} z^{-\alpha},
\end{array} \quad \alpha=1-2 \beta\right. \\
& (\mathrm{VE})\left\{\begin{array}{l}
k_{V}(z)=\frac{G_{0}}{\Gamma(1-\alpha)} z^{-\alpha}, \\
c_{V}(z)=\frac{\eta_{0}}{\Gamma(1+\alpha)} z^{-\alpha},
\end{array}\right.
\end{aligned}
$$

The stress-strain relations of both models involve the Bessel function and, by imposing the proper boundary conditions the following expressions valid at the top of the lamina are obtained:

$$
\begin{aligned}
& \text { (EV) } \sigma(t)=C_{E}(\beta)\left({ }_{c} D_{0^{+}}^{\beta} \gamma\right)(t) \\
& (\mathrm{VE}) \quad \sigma(t)=C_{V}(\beta)\left({ }_{c} D_{0^{+}}^{\beta} \gamma\right)(t)
\end{aligned}
$$

where the coefficients $C_{E}(\beta)$ and $C_{V}(\beta)$ are defined as:

$$
\begin{aligned}
& C_{E}(\beta)=\frac{G_{0} \Gamma(\beta) 2^{2 \beta-1}}{\Gamma(2-2 \beta) \Gamma(1-\beta)}\left(\frac{\eta_{0} \Gamma(\alpha-1)}{G_{0} \Gamma(\alpha+1)}\right)^{\beta} \\
& C_{V}(\beta)=\frac{G_{0} \Gamma(1-\beta) 2^{1-2 \beta}}{\Gamma(2-2 \beta) \Gamma(\beta)}\left(\frac{\eta_{0} \Gamma(\alpha+1)}{G_{0} \Gamma(\alpha-1)}\right)^{\beta}
\end{aligned}
$$

In addition, in order to provide a mechanical meaning of the fractional viscoelasticity, the introduced mechanical models may be discretized and the obtained discrete mechanical models can be easily solved by a classical modal analysis [19]. Moreover the extension of fractional multiphase viscoelastic model is obtained from the introduced exact mechanical models $[46,47]$.

It is easy to observe that the elastic law between stress and strain history, that is modeled by a perfect spring, is analogous to Ohm law between current $i(t)$ and voltage $v(t)$, which is typical of purely resistive conductors. While the Newtonian law is

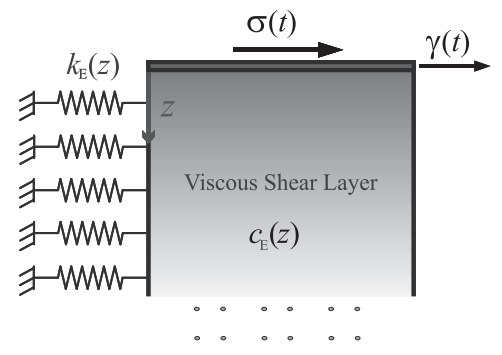

(a) Elasto-Viscous model

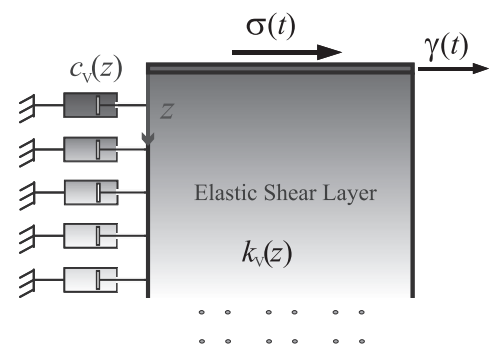

(b) Visco-Elastic model

Fig. 2. Continuous fractional models of viscoelasticity. 
analogous to capacitor law between current and voltage rate $\dot{v}(t)$, which is characteristic of purely capacitive elements. If there are circuits with an electrical behavior intermediate between the purely capacitive and the purely resistive, these result in a fractional current-voltage relation (such as for the stress-strain relation in fractional viscoelasticity); so it is reasonable to think that the viscoelastic analogy and the introduced mechanical model [22,19] may be useful to find a proper fractional electrical model.

\section{Fractional capacitor}

In electrical networks models, the capacitors play a very important role since they model the conservative part of the electric field effects. The well-known Curie's law [48] reveals an empiric relation between the current $i(t)$ related to the applied voltage $v(t)=H(t) \cdot V$ (being $H(t)$ the unit step function):

$$
i(t)=\frac{v(t) t^{-\beta}}{c(\beta) \Gamma(1-\beta)} 0<\beta<1
$$

while $c(\beta)$ is a constant depending on the physical characteristics of the capacitor. Both $c(\beta)$ and $\beta$ can be obtained by experimental data and subsequent best fitting procedure. Eq. (11) may be interpreted as the relaxation function in the following sense. When $v(t)$ is applied to the capacitor, the corresponding $i(t)$ decay with a power law then it relaxes. The current related to $V=1$ is denoted as $G_{E}(t)$, i.e. this current is due to the unit step voltage:

$$
\begin{array}{ll}
G_{E}(t)=\frac{t^{-\beta}}{c(\beta) \Gamma(1-\beta)} ; & t \geqslant 0,0<\beta<1 \\
G_{E}(t)=0 ; & t<0,0<\beta<1
\end{array}
$$

Such a function will be named the 'E-Relaxation Function' (ERF), where the prefix $E$ stands for 'electrical' in order to avoid confusion with the 'Relaxation Function' in viscoelasticity. By using the Boltzmann superposition principle, the current history in the capacitor due to a voltage $v(t)$ can be written as:

$$
i(t)=\int_{0}^{t} G_{E}(t-\bar{t}) \frac{d v(\bar{t})}{d \bar{t}} d \bar{t}
$$

This is a convolution integral whose kernel is the ERF. If the power law kernel Eq. (12) is inserted, the following relation is obtained:

$$
i(t)=\frac{1}{c(\beta) \Gamma(1-\beta)} \int_{0}^{t}(t-\bar{t})^{-\beta} \frac{d v(\bar{t})}{d \bar{t}} d \bar{t}=\frac{1}{c(\beta)}\left({ }^{c} D_{0^{+}}^{\beta} v\right)(t)
$$

It has to be observed that for $\beta=1$ relation Eq. (14) restores an ideal capacitor of capacitance $C=1 / c(1)$; when $\beta=0$ a pure resistor of resistance $R=c(0)$, is obtained. Inside the interval $0<\beta<1$ the fractional capacitor exhibits an intermediate behavior between an ideal resistor and an ideal capacitor. Lets now suppose that a step current $i(t)=H(t) \cdot I$ feeds the capacitor; the voltage related to $I=1$ is denoted as $J_{E}(t)$, i.e. this voltage is due to the unit step current, and will be named as the 'E-Creep Function' (ECF) as the analogous in viscoelasticity as well. By using again the Boltzmann superposition principle, the following relation holds:

$$
v(t)=\int_{0}^{t} J_{E}(t-\bar{t}) \frac{d i(\bar{t})}{d \bar{t}} d \bar{t}
$$

The application of the Laplace transform to Eqs. (13) and (15), enables to straightforwardly observe that ERF and ECF are related each other by the following fundamental relationship:

$$
\widehat{J}_{E}(s) \widehat{G}_{E}(s)=\frac{1}{s^{2}}
$$

where $\widehat{J}_{E}(s)$ and $\widehat{G}_{E}(s)$ are the Laplace transform of $J_{E}(t)$ and $G_{E}(t)$, respectively. By using Eq. (16), the ECF in time domain is easily found, namely:

$$
\begin{aligned}
& J_{E}(t)=\frac{c(\beta) t^{\beta}}{\Gamma(1+\beta)}, \quad t \geqslant 0 \\
& J_{E}(t)=0, \quad t<0
\end{aligned}
$$

By inserting Eq. (17) in Eq. (15), the following relation holds:

$$
v(t)=\frac{c(\beta)}{\Gamma(1+\beta)} \int_{0}^{t}(t-\bar{t})^{\beta} \frac{d i(\bar{t})}{d \bar{t}} d \bar{t}
$$

After some trivial manipulations of the previous equation, it follows:

$$
v(t)=\frac{c(\beta)}{\Gamma(1+\beta)} \int_{0}^{t}(t-\bar{t})^{1-\beta} i(\bar{t}) d \bar{t}=c(\beta)\left(I_{0^{+}}^{\beta} i\right)(t)
$$


where $\left(I_{0^{+}}^{\beta}\right)(t)$ is the Riemann-Liouville fractional integral. Eqs. (14) and (19) are valid for virgin capacitors with $i(0)=0$ and $v(0)=0$. When the initial conditions are different from zero (i.e. $i_{0}$ and $v_{0}$ ), then the terms $v_{0} G_{E}(t)$ and $i_{0} J_{E}(t)$ have to be added in Eqs. (14) and (19), respectively. Power law description of $J_{E}(t)$ or $G_{E}(t)$ leads to conclude that the fractional capacitor exhibits a long-tail memory in the sense that the actual state depends on the entire past history [49,50]. On the contrary, pure resistor has not memory and pure capacitor shows short-time memory. So, in order to distinguish the behavior of fractional capacitor it is named as hereditary capacitor. In the next section the electrical equivalent circuit of Eq. (14) will be presented.

\section{Electrical equivalent circuit of fractional capacitor $(\beta=1 / 2)$}

In this section electrical circuit models of fractional element whose constitutive law is expressed by Eq. (14), is presented for $\beta=1 / 2$. Firstly, let consider the electrical circuit shown in Fig. 3. A longitudinal pure resistor with a per-unit length resistance $r$ and a transversal pure capacitor with a per-unit length capacitance $c$, constitute the elementary cell of a transmission line model along the $x$ abscissa. By using the Kirchhoff voltage and current laws the following coupled partial differential equations hold:

$$
\frac{\partial i(x, t)}{\partial x}=-c \frac{\partial v(x, t)}{\partial t}
$$

and

$$
\frac{\partial v(x, t)}{\partial x}=-r i(x, t)
$$

By combining Eqs. (20) and (21) the following diffusion equation holds:

$$
\frac{\partial^{2} v(x, t)}{\partial x^{2}}-r c \frac{\partial v(x, t)}{\partial t}=0
$$

By Laplace transforming Eq. (22), the following relation holds in the s-domain:

$$
\frac{\partial^{2} \widehat{v}(x, s)}{\partial x^{2}}-r c s \widehat{v}(x, s)=0
$$

The solution of Eq. (23) is expressed as:

$$
\widehat{v}(x, s)=B_{1} e^{x \sqrt{r c s}}+B_{2} e^{-x \sqrt{r c s}}
$$

being $B_{1}$ and $B_{2}$ integration constant to be determined. By imposing the following boundary conditions:

$$
\begin{gathered}
\lim _{x \rightarrow 0} \widehat{v}(x, s)=\widehat{v}(0, s)=\widehat{V}(s) \\
\lim _{x \rightarrow \infty} \widehat{v}(x, s)=0
\end{gathered}
$$

with $\widehat{V}(s)$ as the Laplace transform of the source voltage $v(t)$ placed at $x=0, B_{1}=0$ and $B_{2}=\widehat{V}(s)$ result. So the solution of Eq. (23), become:

$$
\widehat{v}(x, s)=\widehat{V}(s) e^{-x \sqrt{r c s}}
$$

By differentiating with respect to $x$ and taking into account Eq. (21), the following relation holds:

$$
\frac{\partial \widehat{v}(x, s)}{\partial x}=-\widehat{V}(s) \sqrt{r c s} e^{-x \sqrt{r c s}}=-\widehat{r i}(x, s)
$$

that for $x=0$ gives:

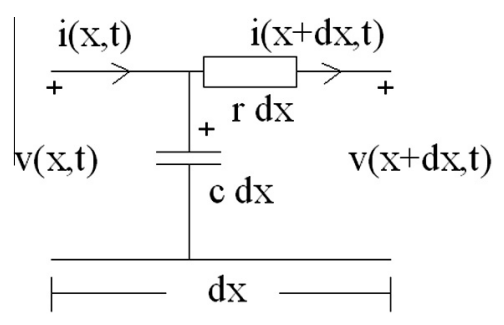

Fig. 3. C-R electrical equivalent circuit for $\beta=1 / 2$. 


$$
\widehat{V}(s) \sqrt{r C S}=\widehat{r i}(0, s)=r \widehat{I}(s)
$$

Eq. (28) is the Laplace transform of the constitutive law relating the external voltage and the corresponding current at $x=0$. The inverse Laplace transform gives:

$$
i(t)=\sqrt{\frac{c}{r}}\left({ }_{c} D_{0^{+}}^{1 / 2} v\right)(t)
$$

By Eq. (29) it is shown that the electrical circuit of Fig. 3 is the exact electrical model of the fractional capacitor with order $\beta=1 / 2$. It is straightforward to obtain the same result by using the circuit model shown in Fig. 4 , in which the roles of resistor and capacitor are exchanged. The following relations hold:

$$
\frac{\partial v(x, t)}{\partial x}=-\frac{1}{c} \int_{0}^{t} i(x, \tau) d \tau
$$

and

$$
\frac{\partial i(x, t)}{\partial x}=-\frac{1}{r} v(x, t)
$$

By combining Eqs. (30) and (31) the following relation holds:

$$
\frac{\partial^{2} v(x, t)}{\partial x^{2}}=\frac{1}{r c} \int_{0}^{t} v(x, \tau) d \tau
$$

and by taking the Laplace transform of (32):

$$
\frac{\partial^{2} \widehat{v}(x, s)}{\partial x^{2}}-\frac{1}{r c s} \widehat{v}(x, s)=0
$$

The solution of the previous equation is:

$$
\widehat{v}(x, s)=B_{1} e^{\frac{x}{\sqrt{r c s}}}+B_{2} e^{\frac{-x}{\sqrt{r c s}}}
$$

The relevant boundary conditions are those in Eq. (25), leading to $B_{1}=0$ and $B_{2}=\widehat{V}(s)$. By differentiating with respect to $x$ and taking into account the Laplace transform of Eq. (30), the current solution is obtained:

$$
\widehat{i}(x, s)=\operatorname{cs} \widehat{V}(s) \frac{1}{\sqrt{r c s}} e^{\frac{-x}{\sqrt{r c s}}}
$$

that in $x=0$ gives (cfr. Eq. 28):

$$
\widehat{i}(0, s)=\widehat{V}(s) \sqrt{\frac{c s}{r}}
$$

Inverse Laplace transform of Eq. (36) gives the same Eq. (29). Then it is possible to conclude that both the electrical equivalent circuits of Figs. 3 and 4, exactly model the constitutive law expressed by Eq. (14).

\section{Discretization}

Electrical circuit of Fig. 3 can be discretized by considering small abscissa intervals $\Delta x$, as shown in Fig. 5. Let denote with $v_{s}=v_{0}$ the source voltage and with $i_{0}$ its current; with $i_{1}, \ldots, i_{n}, \ldots$ the current flowing in the longitudinal resistors $r \Delta x$, and with $v_{1}, \ldots, v_{n-1}, \cdots, v_{n}=0$ the nodal voltages which are the same of that applied to the transversal capacitors $c \Delta x$, except for the last one. By using the constitutive relations of the lumped elements and the Kirchhoff laws, and by omitting the temporal dependence, the following relations hold:

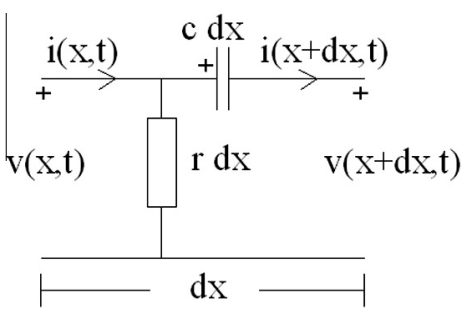

Fig. 4. R-C electrical equivalent circuit for $\beta=1 / 2$. 


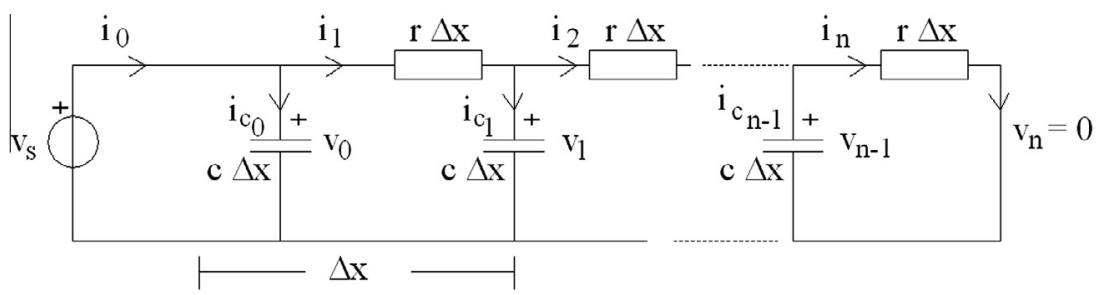

Fig. 5. Discretized model of electrical circuit shown in Fig. 3, driven by a voltage source.

$$
\begin{aligned}
i_{0} & =i_{c_{0}}+i_{1} \\
i_{1} & =\frac{1}{r \Delta x}\left(v_{s}-v_{1}\right) \\
i_{2} & =\frac{1}{r \Delta x}\left(v_{1}-v_{2}\right) \\
\vdots & \vdots \quad \vdots \quad \vdots \\
i_{n-1} & =\frac{1}{r \Delta x}\left(v_{n-2}-v_{n-1}\right) \\
i_{n} & =\frac{1}{r \Delta x} v_{n-1}
\end{aligned}
$$

and

$$
\begin{gathered}
i_{c_{0}}=c \Delta x \dot{v}_{0} \\
i_{c_{1}}=i_{1}-i_{2}=c \Delta x \dot{v}_{1} \\
i_{c_{2}}=i_{2}-i_{3}=c \Delta x \dot{v}_{2} \\
\vdots \quad \vdots \quad \vdots \\
i_{c_{n-1}}=i_{n-1}-i_{n}=c \Delta x \dot{v}_{n-1}
\end{gathered}
$$

When $n$ increases, the currents decrease and then a truncation can be performed. Let now introduce the node voltage matrix $\boldsymbol{v}^{T}=\left[\begin{array}{llll}v_{1} & \cdots & v_{n-1}\end{array}\right]$ and the matrix of the corresponding node voltage temporal derivatives $\dot{\boldsymbol{v}}^{T}=\left[\begin{array}{llll}\dot{v}_{1} & \cdots & \dot{v}_{n-1}\end{array}\right]$; by using Eqs. (37) to obtain the temporal derivatives of node voltages of Eqs. (38), the following relation holds:

$$
\dot{\boldsymbol{v}}=\boldsymbol{A}_{v} \boldsymbol{v}+\mu v_{s} \boldsymbol{C}_{1}
$$

where $v_{s}$ is the source voltage, $\mu=1 /\left[\operatorname{rc}(\Delta x)^{2}\right], \mathbf{C}_{1}^{T}=\left[\begin{array}{llll}1 & 0 & \cdots & 0\end{array}\right]$, and $\mathbf{A}_{v}$ is the following tridiagonal matrix [51,52]:

$$
\mathbf{A}_{v}=-\mu\left[\begin{array}{cccccc}
2 & -1 & 0 & 0 & \ldots & 0 \\
-1 & 2 & -1 & 0 & \ldots & 0 \\
0 & -1 & 2 & -1 & \ldots & 0 \\
\vdots & \ldots & \vdots & \ldots & \vdots & \ldots \\
0 & \cdots & 0 & -1 & 2 & -1 \\
0 & \cdots & 0 & 0 & -1 & 2
\end{array}\right]
$$

Let indicate with $\lambda_{j}$ the eigenvalues of matrix $\frac{-1}{\mu} \mathbf{A}_{v}$ and with $\phi_{j}$ the corresponding eigenvectors normalized with the identity matrix. $\phi_{k, j}$ indicates the $k$ th component of the vector $\boldsymbol{\phi}_{j}$. Since $\mathbf{A}_{v}$ is symmetric (and positive as definition), the matrix $\boldsymbol{\phi}$ whose $j$ th column is the eigenvector $\phi_{j}$ leads to the following relations:

$$
\begin{gathered}
\boldsymbol{\phi}^{T}=\boldsymbol{\phi} \\
\boldsymbol{\phi}^{T} \mathbf{A}_{\nu} \boldsymbol{\phi}=-\mu \boldsymbol{\Lambda}
\end{gathered}
$$

where $\boldsymbol{\Lambda}$ is the diagonal matrix whose $j$ th element is $\lambda_{j}$. By performing the following coordinates transform:

$$
\mathbf{y}=\phi \mathbf{v}
$$

in Eq. (39), and multiplying on the left by $\phi^{T}$, the following relation is obtained:

$$
\dot{\mathbf{y}}=-\mu \mathbf{\Lambda} \mathbf{y}+\mu v_{s} \mathbf{u}
$$

where $\mathbf{u}^{T}$ is the first row of the matrix $\phi$, namely: 


$$
\mathbf{u}=\boldsymbol{\phi}^{T} \mathbf{C}_{1}=\left[\phi_{11} \phi_{12} \ldots \phi_{1 n}\right]
$$

From Eq. (43) it results that in the modal space $\mathbf{y}$ the differential equations are decoupled and are given in the form:

$$
\dot{y}_{j}(t)+\mu \lambda_{j} y_{j}(t)=\mu \phi_{1 j} v_{s}(t)
$$

and the corresponding electrical equivalent circuit is simply that shown in Fig. 6. The response of the jth electrical circuit is given as:

$$
y_{j}(t)=y_{j}(0) e^{-\rho_{j} t}+\mu \phi_{1 j} \int_{0}^{t} e^{-\rho_{j}(t-\bar{t})} v_{s}(\bar{t}) d \bar{t}
$$

where $y_{j}(0)=\phi_{j}^{T} \mathbf{v}(0), \rho_{j}=\mu \lambda_{j}$ and $v_{s}(t)$ is the source voltage. Once all the responses $y_{j}(t)$ are evaluated, the voltage vector $\mathbf{v}$ is readily formed as:

$$
\mathbf{v}=\phi^{T} \mathbf{y}
$$

and the current $i(t)$ is then computed by the first of Eqs. (37). From Fig. 6 it appears the multi-scale physical significance of the fractional constitutive law.

Let now suppose that the circuit of Fig. 5 is driven by a current source, as reported in Fig. 7. By using the same notation as in the previous case, the following equations hold:

$$
\begin{gathered}
i_{1}=\frac{1}{r \Delta x}\left(v_{0}-v_{1}\right) \\
i_{2}=\frac{1}{r \Delta x}\left(v_{1}-v_{2}\right) \\
\vdots \quad \vdots \quad \vdots \quad \vdots \\
i_{n-1}=\frac{1}{r \Delta x}\left(v_{n-2}-v_{n-1}\right) \\
i_{n}=\frac{1}{r \Delta x} v_{n-1}
\end{gathered}
$$

and

$$
\begin{gathered}
i_{c_{0}}=i_{s}-i_{1}=c \Delta x \dot{v}_{0} \\
i_{c_{1}}=i_{1}-i_{2}=c \Delta x \dot{v}_{1} \\
i_{c_{2}}=i_{2}-i_{3}=c \Delta x \dot{v}_{2} \\
\vdots \quad \vdots \quad \vdots \\
i_{c_{n-1}}=i_{n-1}-i_{n}=c \Delta x \dot{v}_{n-1}
\end{gathered}
$$

Let now introduce the node voltage $\mathbf{v}^{T}=\left[\begin{array}{lll}v_{0} & \cdots & v_{n-1}\end{array}\right]$ and the matrix of the corresponding node voltage temporal derivatives $\dot{\mathbf{v}}^{T}=\left[\begin{array}{llll}\dot{v}_{0} & \cdots & \dot{v}_{n-1}\end{array}\right]$; after some trivial manipulations the following relation holds:

$$
\dot{\mathbf{v}}=\mathbf{A}_{i} \mathbf{v}+\frac{i_{s}}{c \Delta x} \mathbf{C}_{1}
$$

where $\mathbf{A}_{i}$ is the following tridiagonal matrix:

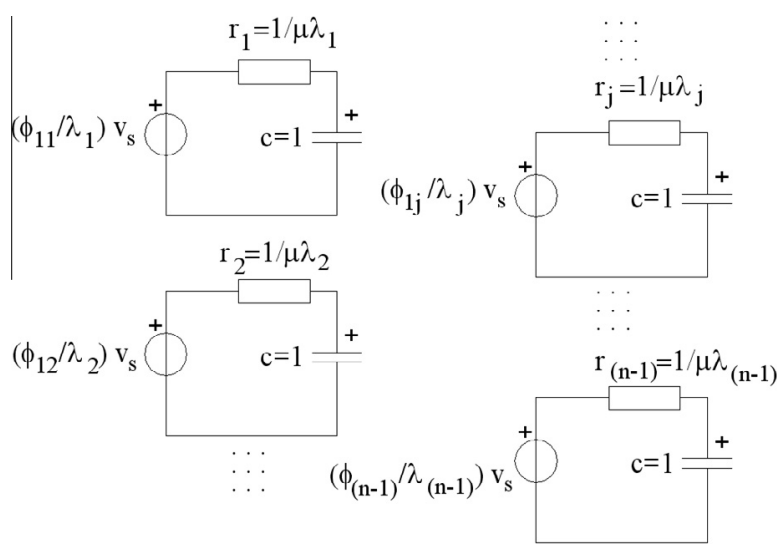

Fig. 6. Electrical circuit of Fig. 5 in the modal space. 


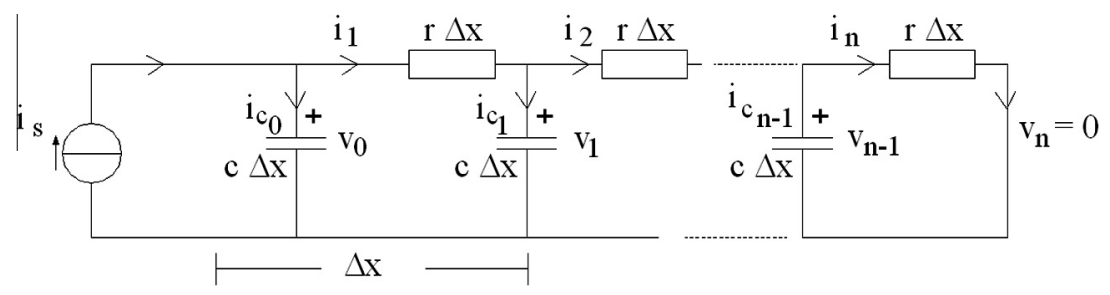

Fig. 7. Discretized model of electrical circuit shown in Fig. 3, driven by a current source.

$$
\mathbf{A}_{i}=-\mu\left[\begin{array}{cccccc}
1 & -1 & 0 & 0 & \ldots & 0 \\
-1 & 2 & -1 & 0 & \ldots & 0 \\
0 & -1 & 2 & -1 & \cdots & 0 \\
\vdots & \ldots & \vdots & \ldots & \vdots & \ldots \\
0 & \ldots & 0 & -1 & 2 & -1 \\
0 & \ldots & 0 & 0 & -1 & 2
\end{array}\right]
$$

The eigenvalues $\lambda_{j}$ of matrix $\frac{-1}{\mu} \mathbf{A}_{i}$ and the corresponding eigenvectors $\phi_{j}$ normalized with the identity matrix, are:

$$
\begin{aligned}
& \lambda_{j}=2-2 \cos \left(\frac{2 j-1}{2 n+1} \pi\right), \quad j=1,2, \ldots, n \\
& \phi_{k, j}=\sqrt{\frac{4}{2 n+1}} \cos \left[\frac{(2 j-1)(2 k-1) \pi}{2(2 n+1)}\right], \quad j, k=1,2, \ldots, n
\end{aligned}
$$

where $\phi_{k, j}$ is the $k$ th component of the vector $\phi_{j}$.

By using the same transformation as in Eq. (41) and Eq. (42), the following relation similar to that reported in Eq. (43), is obtained:

$$
\dot{\boldsymbol{y}}=-\mu \boldsymbol{\Lambda} \boldsymbol{y}+\frac{i_{s}}{c \Delta x} \mathbf{u}
$$

where $\mathbf{u}^{T}$ is reported in Eq. (44). From Eq. (53) it results that in the modal space $\mathbf{y}$ the differential equations are decoupled and are given in the form:

$$
\dot{y}_{j}(t)+\mu \lambda_{j} y_{j}(t)=\frac{\phi_{1 j} i_{s}}{c \Delta x}
$$

and so:

$$
y_{j}(t)=y_{j}(0) e^{-\rho_{j} t}+\frac{\phi_{1 j}}{c \Delta x} \int_{0}^{t} e^{-\rho_{j}(t-\bar{t})} i_{s}(\bar{t}) d \bar{t}
$$

A similar result may also be achieved from the electrical equivalent circuit of Fig. 4, by using the discretized circuit reported in Fig. 8. The following relations hold:

$$
\begin{gathered}
i_{1}=c \Delta x\left(\dot{v}_{s}-\dot{v}_{1}\right) \\
i_{2}=c \Delta x\left(\dot{v}_{1}-\dot{v}_{2}\right) \\
\vdots \quad \vdots \quad \vdots \\
i_{n-1}=c \Delta x\left(\dot{v}_{n-2}-\dot{v}_{n-1}\right) \\
i_{n}=c \Delta x \dot{v}_{n-1}
\end{gathered}
$$

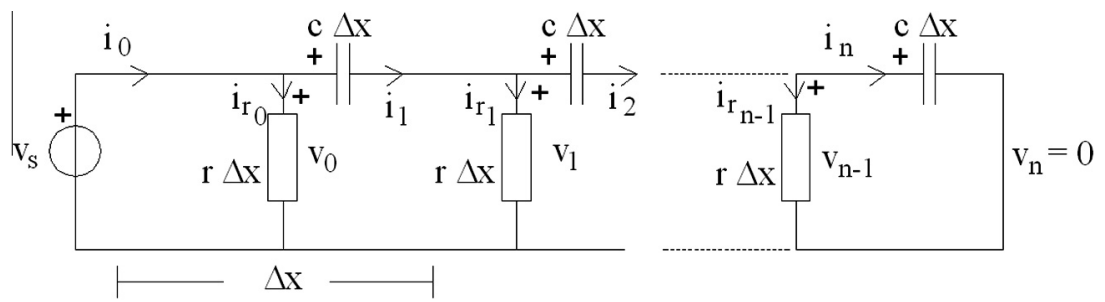

Fig. 8. Discretized model of electrical circuit shown in Fig. 4, driven by a voltage source. 
and

$$
\begin{gathered}
i_{r_{0}}=i_{0}-i_{1}=\frac{v_{s}}{r \Delta x} \\
i_{r_{1}}=i_{1}-i_{2}=\frac{v_{1}}{r \Delta x} \\
\vdots \quad \vdots \quad \vdots \quad \vdots \\
i_{r_{n-1}}=i_{n-1}-i_{n}=\frac{v_{n-1}}{r \Delta x}
\end{gathered}
$$

By using the node voltage matrix $\mathbf{v}^{T}=\left[v_{1} \ldots v_{n-1}\right]$ and the matrix of the corresponding node voltage temporal derivatives $\dot{\mathbf{v}}^{T}=\left[\begin{array}{llll}\dot{v}_{1} & \cdots & \dot{v}_{n-1}\end{array}\right]$, the differential equations are given in the form:

$$
\mathbf{A}_{v} \dot{\mathbf{v}}=\mu^{2} \mathbf{v}-\mu \dot{v}_{s} \mathbf{C}_{1}
$$

where $\mathbf{A}_{v}$ has been already defined in Eq. (40). By using the previous procedure, the coordinate transform leads to the following set of decoupled differential equations:

$$
\dot{\mathbf{y}}=-\mu \boldsymbol{\Lambda}^{-1} \mathbf{y}-\dot{v}_{s} \boldsymbol{\Lambda}^{-1} \mathbf{u}
$$

where $\mathbf{u}$ is defined in Eq. (44), and whose $j$ th equation is:

$$
\dot{y}_{j}(t)+\frac{\mu}{\lambda_{j}} y_{j}(t)=-\frac{\phi_{1 j}}{\lambda_{j}} \dot{v}_{s}(t)
$$

The solution of Eq. (60) is given as:

$$
y_{j}(t)=y_{j}(0) e^{-\frac{\mu}{\lambda_{j}} t}-\frac{\phi_{1 j}}{\lambda_{j}} \int_{0}^{t} e^{-\frac{\mu}{\gamma_{j}}(t-\bar{t})} \dot{v}_{s}(\bar{t}) d \bar{t}
$$

The electrical circuit in the modal space is shown in Fig. 9.

Let now suppose that the circuit of Fig. 8 is driven by a current source, as reported in Fig. 10. The following relations hold:

$$
\begin{gathered}
i_{1}=c \Delta x\left(\dot{v}_{0}-\dot{v}_{1}\right) \\
i_{2}=c \Delta x\left(\dot{v}_{1}-\dot{v}_{2}\right) \\
\vdots \quad \vdots \quad \vdots \\
i_{n-1}=c \Delta x\left(\dot{v}_{n-2}-\dot{v}_{n-1}\right) \\
i_{n}=c \Delta x \dot{v}_{n-1}
\end{gathered}
$$

and

$$
\begin{gathered}
i_{r_{0}}=i_{s}-i_{1}=\frac{v_{0}}{r \Delta x} \\
i_{r_{1}}=i_{1}-i_{2}=\frac{\nu_{1}}{r \Delta x} \\
\vdots \quad \vdots \quad \vdots \\
i_{r_{n-1}}=i_{n-1}-i_{n}=\frac{v_{n-1}}{r \Delta x}
\end{gathered}
$$

By using the node voltage matrix $\mathbf{v}^{T}=\left[v_{0} \cdots v_{n-1}\right]$ and the matrix of the corresponding node voltage temporal derivatives $\dot{\mathbf{v}}^{T}=\left[\begin{array}{lll}\dot{v}_{0} & \cdots & \dot{v}_{n-1}\end{array}\right]$, after some trivial manipulations the following differential equations holds:

$$
\mathbf{A}_{i} \dot{\mathbf{v}}=\mu^{2} \mathbf{v}-\frac{\mu}{c \Delta x} i_{s} \mathbf{C}_{1}
$$

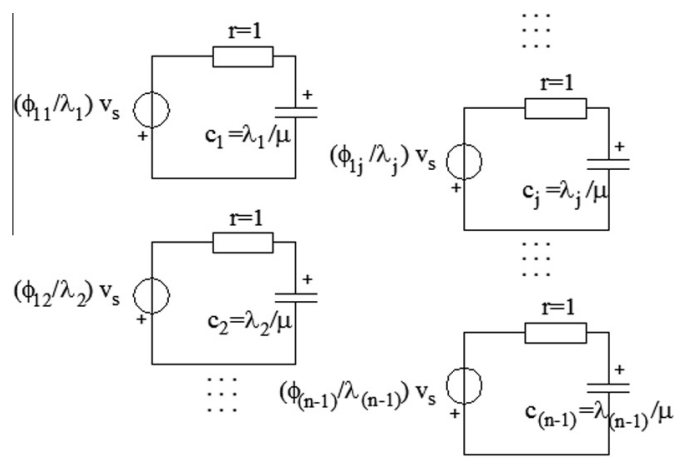

Fig. 9. Electrical circuit of Fig. 8 in the modal space. 


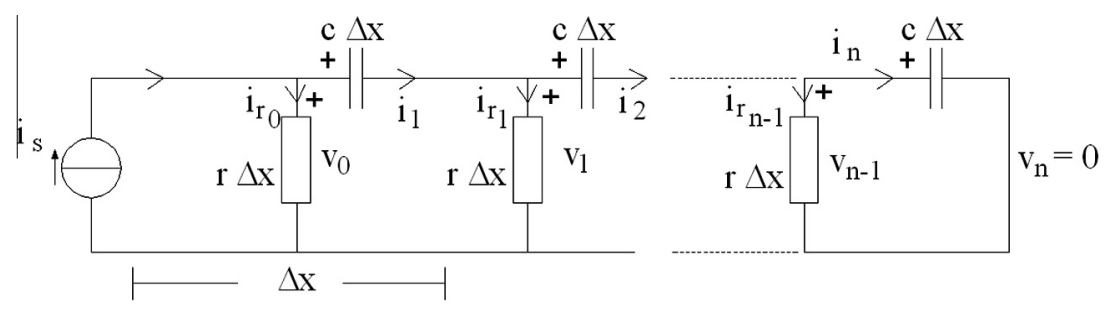

Fig. 10. Discretized model of electrical circuit shown in Fig. 4, driven by a current source.

where $\mathbf{A}_{i}$ has been already defined in Eq. (51). By using the previous procedure, the coordinate transform leads to the following set of decoupled differential equations:

$$
\dot{\mathbf{y}}=-\mu \boldsymbol{\Lambda}^{-1} \mathbf{y}-\frac{i_{s}}{c \Delta x} \Lambda^{-1} \mathbf{u}
$$

where $\mathbf{u}^{T}$ is reported in Eq. (44), and whose $j$ th equation is:

$$
\dot{y}_{j}(t)+\frac{\mu}{\lambda_{j}} y_{j}(t)=-\frac{\phi_{1 j}}{\lambda_{j}} \frac{i_{s}}{c \Delta x}
$$

The solution of Eq. (66) is given as:

$$
y_{j}(t)=y_{j}(0) e^{-\frac{\mu}{\lambda_{j}} t}-\frac{\phi_{1 j}}{c \Delta x \lambda_{j}} \int_{0}^{t} e^{-\frac{\mu}{\lambda_{j}}(t-\bar{t})} i_{s}(\bar{t}) d t
$$

\section{Numerical examples}

In this section numerical examples are presented in order to show the accuracy of the discretized model. At first, for the discretized circuit shown in Fig. 5, the current response $i_{0}$ to a unit step voltage $v_{s}(t)=H(t)$ is considered. In this case, the exact solution of Eq. (13) is the ERF described in Eq. (12). Different comparisons have been carried out. In Fig. 11 the comparison between discrete circuit results, with 2000 eigenvalues, $L=2 \mathrm{~m}$, and ERF function behavior described in Eq. (12), is reported. In order to better compare the discretized model based on modal analysis, another example is reported in Fig. 12, related to the same circuit. Moreover, finite difference time domain (FDTD) method [53-55] is also used in order to numerically solve the coupled Eqs. (20) and (21). In Fig. 13, for the same example reported in Fig. 12, FDTD results are compared with analytical ones. A very good agreement has been reached.

Moreover, the voltage response $v_{0}$ to a unit step current $i_{s}(t)=H(t)$ is also considered. The discrete circuit shown in Fig. 7, is used. The exact solution of Eq. (15) for the unit step function is the creep function described in Eq. (17). In Fig. 14 the comparison between modal analysis results and ECF profile reported in Eq. (17), is shown. FDTD simulations have been carried out also in this case. In Figs. 15 and 16 short and long time simulation results are compared withe ECF profile, respectively. As reported in [19], discrete model is able to represent the behavior of $v_{0}$ voltage only for a certain time interval.

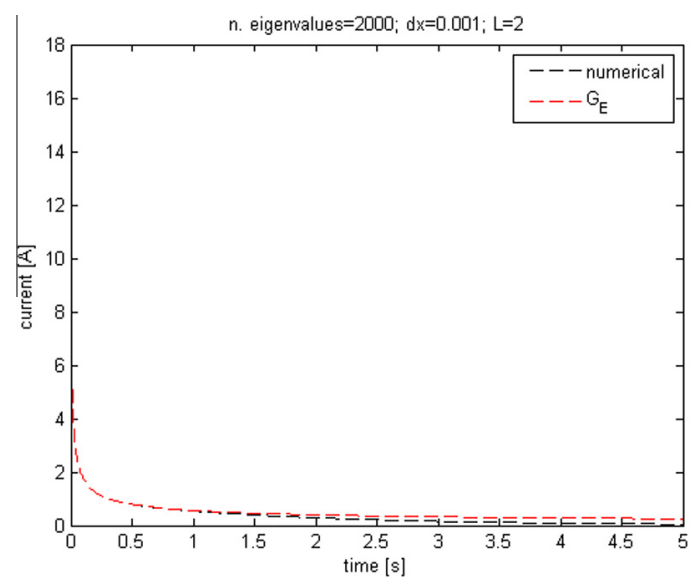

Fig. 11. Comparison between discrete circuit modal analysis of Fig. 5 results, and ERF function behavior described in Eq. (12). 


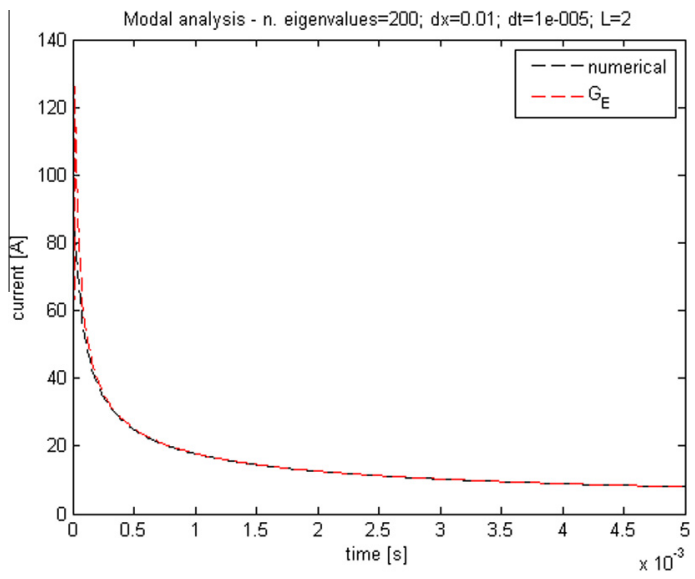

Fig. 12. Comparison between discrete circuit modal analysis of Fig. 5 results, and ERF function behavior described in Eq. (12).

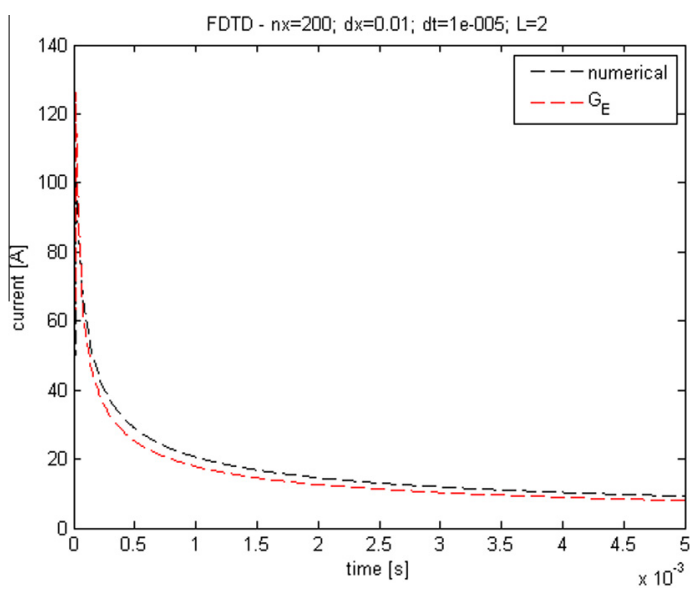

Fig. 13. Comparison between FDTD method results and ERF function behavior described in Eq. (12).

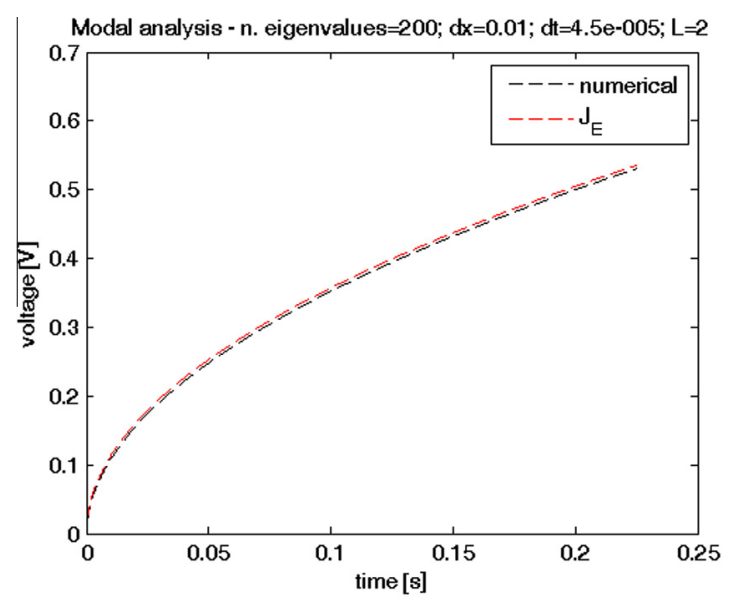

Fig. 14. Comparison between modal analysis results and ECF profile: nodal voltage $v_{0}$ of Fig. 7 . 


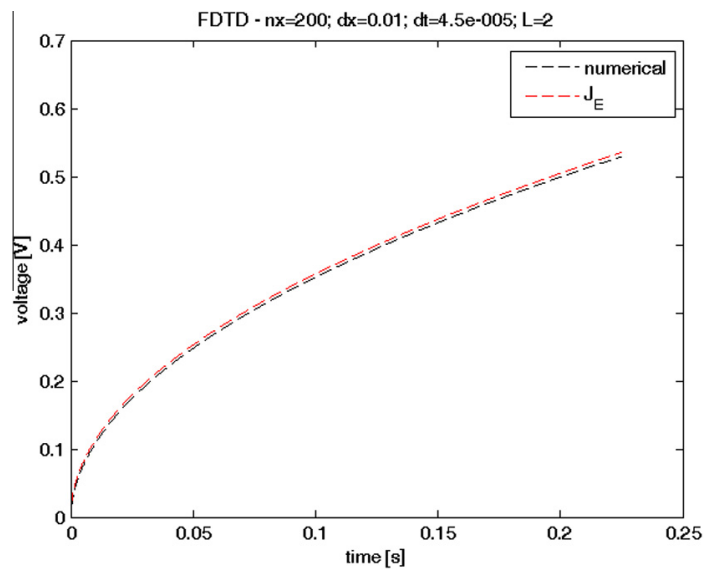

Fig. 15. FDTD results compared with ECF profile: short time simulation.

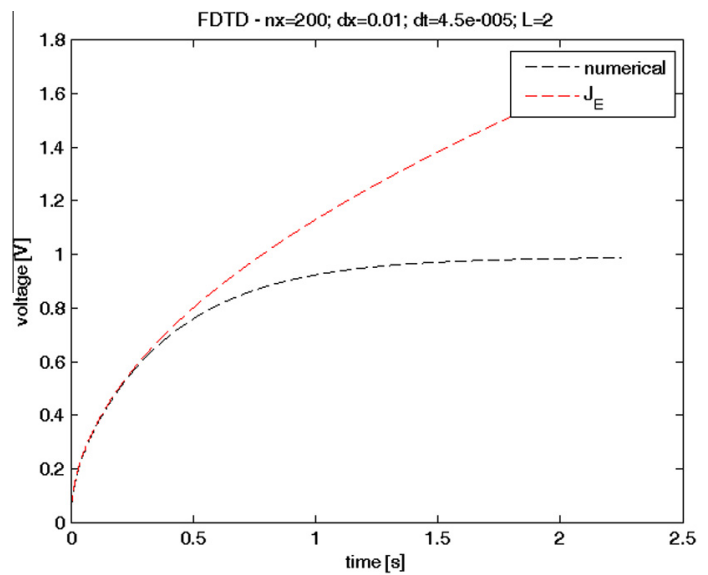

Fig. 16. FDTD results compared with ECF profile: long time simulation.

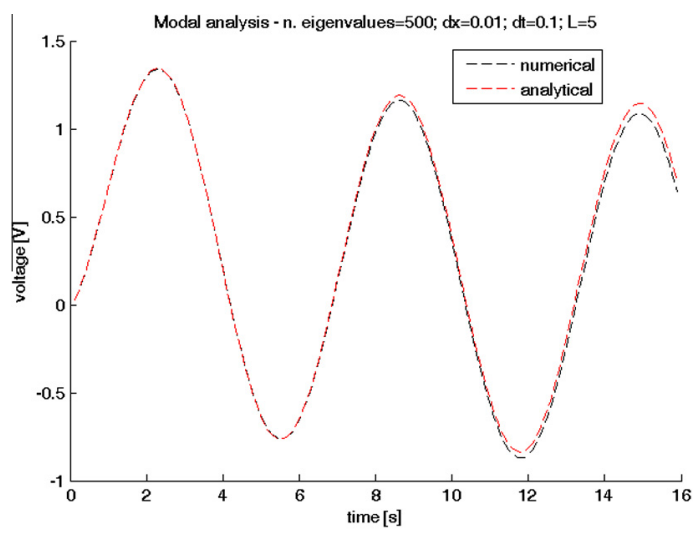

Fig. 17. $v_{0}$ voltage time profile of Fig. 7 with a sinusoidal input: $\omega=1 \mathrm{rad} / \mathrm{s}$. The input amplitude is set to a unit value. 
In Fig. $17 v_{0}$ voltage time profile of Fig. 7 with a sinusoidal current source with $\omega=1 \mathrm{rad} / \mathrm{s}$ and unit amplitude is reported, compared with the analytical one. A $5 \mathrm{~m}$ length configuration has been selected.

The exact response to such an input for Eq. (15), is:

$$
v(t)=\frac{\bar{i}_{s} \omega t^{1+\beta}}{c(\beta) \Gamma(2+\beta)}{ }_{1} F_{2}\left[1 ; \frac{2+\beta}{2}, \frac{3+\beta}{2} ;-\left(\frac{\omega t^{2}}{2}\right)\right]
$$

where ${ }_{1} F_{2}(\cdot)$ is the hypergeometric function [56] that is defined as

$$
{ }_{p} F_{q}\left(a_{1}, \ldots, a_{p} ; b_{1}, \ldots, b_{q} ; z\right)=\sum_{n=0}^{\infty} \frac{\left(a_{1}\right)_{k}\left(a_{2}\right)_{k} \ldots\left(a_{p}\right)_{k}}{\left(b_{1}\right)_{k}\left(b_{2}\right)_{k} \ldots\left(b_{q}\right)_{k}} \frac{z^{k}}{k !}
$$

where $(a)_{k}=k(a+1) \ldots(a+k-1)$ is the Pochhammer Symbol. For $\beta=1 / 2$ Eq. (68) becomes:

$$
v(t)=\frac{\bar{i}_{s} \omega t^{3 / 2}}{c(1 / 2) \Gamma(5 / 2)}{ }_{1} F_{2}\left[1 ; \frac{5}{4}, \frac{7}{4} ;-\left(\frac{\omega t^{2}}{2}\right)\right] .
$$

As shown in Fig. 17, a very good agreement has been obtained.

\section{Conclusions}

Electrical analogous models of fractional hereditary materials have been introduced. At first, mechanical models of materials viscoelasticity behavior have been approached by using fractional calculus. By combining springs and dashpots, different viscoelastic models have been obtained. These models have been equivalently modeled as electrical circuits, where the spring and dashpot are analogous to the capacitance and resistance, respectively. The proposed models have been validated by using modal analysis. The comparison with numerical results obtained also by using FDTD method shows that, for long time simulations, the correct time behavior can be revealed only with modal analysis. The use of electrical analogous in viscoelasticity can help to better investigate the real behavior of fractional hereditary materials.

\section{Acknowledgment}

Support by Universita' degli Studi di Palermo is gratefully acknowledged.

\section{References}

[1] Sakthivel R, Ganesh R, Ren Y, Anthoni SM. Approximate controllability of nonlinear fractional dynamical systems. Commun Nonlinear Sci Numer Simul 2013;18(12):3498-508.

[2] Yang Z, Cao J. Initial value problems for arbitrary order fractional differential equations with delay. Commun Nonlinear Sci Numer Simul 2013;18(11):2993-3005.

[3] Liu Y. Existence of solutions for impulsive differential models on half lines involving Caputo fractional derivatives. Commun Nonlinear Sci Numer Simul 2013;18(10):2604-25.

[4] Liu Y. Application of Avery-Peterson fixed point theorem to nonlinear boundary value problem of fractional differential equation with the Caputos derivative. Commun Nonlinear Sci Numer Simul 2012;17(12):4576-84.

[5] Wang J, Dong X, Zhou Y. Analysis of nonlinear integral equations with Erdlyi-Kober fractional operator. Commun Nonlinear Sci Numer Simul 2012;17(8):3129-39.

[6] Machado JT, Kiryakova V, Mainardi F. Recent history of fractional calculus. Commun Nonlinear Sci Numer Simul 2011;16(3):4756-67.

[7] Agrawal Om P, Muslih Sami I, Baleanu D. Generalized variational calculus in terms of multi-parameters fractional derivatives. Commun Nonlinear Sci Numer Simul 2011;16(12):4756-67.

[8] Bouafoura MK, Braiek NB. Controller design for integer and fractional plants using piecewise orthogonal functions. Commun Nonlinear Sci Numer Simul 2010;15(5):1267-78.

[9] Baleanu D, Trujillo JI. A new method of finding the fractional Euler-Lagrange and Hamilton equations within Caputo fractional derivatives. Commun Nonlinear Sci Numer Simul 2010;15(5):1111-5.

[10] Gross B. Electrical analogs for viscoelastic systems. J Polym Sci 1956;20(95):371-80.

[11] Carlson G, Halijak C. Approximation of fractional capacitors $(1 / s)^{(1 / n)}$ by a regular newton process. IEEE Trans Circuit Theory 1964;11(2):210-3.

[12] Watt MM-K, North York C. United states patent 6411494: Distributed capacitor, June; 2002.

[13] Petras I, Sierociuk D, Podlubny I. Identification of parameters of a half-order system. IEEE Trans Signal Process 2012;60(10):55615566.

[14] Podlubny I, Petras I, Vinagre BM, O’Leary P, Dorcak L. Analogue realizations of fractional-order controllers. Nonlinear Dyn 2002;29(14):281296.

[15] Caponetto R, Dongola G, Fortuna L., PetrÅ I. Fractional order systems: modeling and control applications. World Scientific series on nonlinear science, Series A, vol. 72. World Scientific; 2010.

[16] Strukov DB, Snider GS, Stewart DR, Williams RS. The missing memristor found. Nature 2008;453:80-3.

[17] Petras I, Chen YQ, Coopmans C. Fractional-order memristive systems. Proc IEEE Conf Emerg Tech Factory Autom 2009:1-8.

[18] Westerlund S, Ekstam L. Capacitor theory. IEEE Trans Dielectr Electr Insul 1994;1(5):826-39.

[19] Di Paola M, Pinnola FP, Zingales M. A discrete mechanical model of fractional hereditary materials. Meccanica: Int J Theor Appl Mech 2013;48(7):1573-86.

[20] Jerri A. Introduction to integral equations with applications. New York: Wiley; 1999.

[21] Di Paola M, Pirrotta A, Valenza A. Visco-elastic behavior through fractional calculus: an easier method for best fitting experimental results. J Mater Sci Dec 2011;43(12):799-806.

[22] Di Paola M, Zingales M. Exact mechanical models of fractional hereditary materials. J Rheol 2012:56(5):983-1004.

[23] Bates JHT. Lung mechanics. An inverse modeling approach. Cambridge University Press, 2009.

[24] Ionescu CM, Kosinski W, De Keyser R. Viscoelasticity and fractal structure in a model of human lungs. Arch Mech 2010;62(1):21-48. 
[25] Ionescu CM, De Keyser R. Relations between fractional-order model parameters and lung pathology in chronic obstructive pulmonary disease. IEEE Trans Biomed Eng 2009;56(4):978-87.

[26] Craiem DO, Armentano RL. Arterial viscoelasticity: a fractional derivative mode, In: Proceedings of 28th IEEE EMBS annual international conference, Aug 30-Sept 3, New York City, USA; 2009.

[27] Craiem DO, Rojo FJ, Atienza JM, Armentano RL, Guinea GV. Fractional-order viscoelasticity applied to describe uniaxial stress relaxation of human arteries. Phys Med Biol 2008;53(17).

[28] Valdez-Jasso D, Haider MA, Banks HT, Santana DB, German YZ, Armentano RL, Olufsen MS. Analysis of viscoelastic wall properties in ovine arteries. IEEE Trans Biomed Eng 2009;56(2):210-9.

[29] Flügge W. Viscoelasticity. Waltham: Blaisdell Publishing Company; 1967.

[30] Christensen RM. Theory of viscoelasticity. New York: Academic Press; 1982.

[31] Nutting PG. A new general law of deformation. J Franklin Inst May 1921;191:679-85.

[32] Gemant A. A method of analyzing experimental results obtained from elasto-viscous bodies. Physics Aug 1936;7:311-7.

[33] Deseri L, Di Paola M, Zingales M, Pollaci P. Power-law hereditariness of hierarchical fractal bones. Int J Numer Meth Biomed Engng 2013;29(12):1338-60. http://dx.doi.org/10.1002/cnm.2572.

[34] Di Paola M, Fiore V, Pinnola, FP, Valenza A. On the influence of the initial ramp for a correct definition of the parameters of fractional viscoelastic materials. Mech Mater 2014;69(1):63-70. http://dx.doi.org/10.1016/i.mechmat 2013.09.017.

[35] Podlubny I. Fractional differential equations. San Diego: Academic Press; 1999.

[36] Oldham KB, Spainer J. The fractional calculus: theory and applications of differentiation and integration to arbitrary order. New York: Academic Press; 1974.

[37] Caputo M. Linear models of dissipation whose Q is almost frequency independent-II. Geophys J R Astron Soc 1967;13:529-39 (Reprinted recently in: Fract. Calc. Appl. Anal., vol. 11, no 1, p. 3-14, 2008).

[38] Miller KS, Ross B. An introduction to the fractional calculus and fractional differential equations. New York: Wiley-InterScience; 1993.

[39] Samko GS, Kilbas AA, Marichev OI. Fractional integrals and derivatives: theory and applications. New York (NY): Gordon and Breach; 1993.

[40] Kilbas AA, Srivastava HM, Trujillo JJ. Theory and applications of fractional differential equations. Amsterdam: Elsevier; 2006.

[41] Hilfer R. Application of fractional calculus in physics. Singapore: World Scientific; 2000.

[42] Gerasimov AN. A generalization of linear laws of deformation and its application to inner friction problems. Prikl Mat Mekh 1949;12:251-9 (in Russian).

[43] Scott Blair GW, Caffyn JE. An application of the theory of quasi-properties to the treatment of anomalous strain-stress relations. Philos Mag 1949;40(300):80-94

[44] Slonimsky GL. On the law of deformation of highly elastic polymeric bodies. Dokl Akad Nauk SSSR 1961;140(2):343-6 [in Russian].

[45] Mainardi F. Fractional Calculus and Waves in Linear Viscoelasticity. London: Imperial College Press-World Scientific Publishing Co. Pte. Ltd.; 2010.

[46] Di Paola M, Pinnola FP, Zingales M. Fractional multi-pahse hereditary materials: Mellin transform and multi-scale fractances, In: Proceedings of ECCOMAS 2012 - European Congress on Computational Methods in Applied Sciences and Engineering, e-Book Full Papers, p. 4735-45.

[47] Di Paola M, Pinnola FP, Zingales M. Fractional differential equations and related exact mechanical models. Comput Math Appl 66(5): 608-20.

[48] Curie MJ. Recherches sur la conductibilit des corps cristallises. Annales de chimie et de physique 1889;18(6):203-69.

[49] Outstaloup A. La drivation non entire: thorie, synthse et applications. Paris: Herms; 1995.

[50] Jesus IS, Tenreiro Machado JA. Development of fractional order capacitors based on electrolyte processes. Nonlinear Dyn 2009;56:4555.

[51] Podlubny I. Matrix approach to discrete fractional calculus. Fract Calc Appl Anal 2000;3(4):359-86.

[52] Podlubny I, Chechkin AV, Skovranek T, Chen YQ, Vinagre B. Matrix approach to discrete fractional calculus II: partial fractional differential equations. J Comput Phys 2009;228(8):3137-53.

[53] Ala G, Di Silvestre ML, Viola F, Francomano E. Soil ionization due to high pulse transient currents leaked by earth electrodes. Prog Electromagnet Res B 2009;14:1-21.

[54] Ala G, Di Piazza MC, Ragusa A, Viola F, Vitale G. EMI analysis in electrical drives under lightning surge conditions. IEEE Trans Electromagnet Compat 2012;54(4):850-9.

[55] Ala G, Francomano E. A marching-on in time meshless kernel based solver for full-wave electromagnetic simulation. Numer Algorithms 2013;62(4):229-37.

[56] Abramowitz M, Stegun IA. Handbook of mathematical functions. USA: NIST; 1972. 